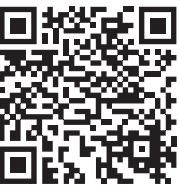

\footnotetext{
Palabras clave:

Entrenamiento

basado en simulación, educación médica, suturas, colgajos locales.

Keywords: Simulation-based training, medical education, sutures, local flaps.
}

\footnotetext{
* Centro de Simulación.

* Escuela de Medicina.

$\S$ Departamento de

Cirugía Digestiva.

I Sección de

Cirugía Plástica

y Reconstructiva,

División de Cirugía.

Pontificia Universidad Católica de Chile.

Santiago, Chile.
}

Recibido: 27/10/2020

Aceptado: 25/11/2020

doi: $10.35366 / 97899$

\title{
Entrenamiento de colgajos locales en un modelo simulado de alta fidelidad y bajo costo
}

\author{
Local flaps training in a high-fidelity and \\ low-cost simulation model
}

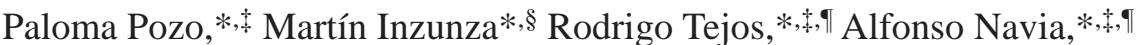 \\ Cristián Jarry, ${ }^{*, \S}$ Pablo Achurra, ${ }^{*, \S}$ Julián Varas, ${ }^{*, \S}$ Claudio Guerra, ${ }^{\ddagger, \llbracket}$ Susana Searle ${ }^{\ddagger, \Uparrow}$
}

\section{RESUMEN}

Introducción: Existen diversos modelos para entrenar colgajos locales; sin embargo, la práctica en superficies convexas ha sido poco explorada. Objetivo: Evaluar el desempeño de alumnos de medicina en la adquisición de técnicas de colgajos locales en un modelo simulado convexo de cuero cabelludo. Material y métodos: Estudio experimental no aleatorizado. Se diseñó un programa de entrenamiento utilizando un modelo de simulación definido a partir de un consenso de expertos. El modelo se confeccionó utilizando piel de cerdo ex vivo sobre un cráneo humano. Se realizaron videograbaciones de cada participante realizando un colgajo de avance y de rotación antes de comenzar el entrenamiento y al terminar el programa. Se asignaron puntajes de desempeño de acuerdo con la escala OSATS. Se compararon las evaluaciones iniciales y finales utilizando estadística no paramétrica. Resultados: Se incluyeron 21 participantes. Todos aumentaron significativamente sus puntajes OSATS al comparar la evaluación inicial con la final tanto en colgajo de avance (15 [13-16] vs 23 [22-24] p < 0.001) como en colgajo de rotación ( 15 [14-17] vs 24 [23-25] p < 0.001). Conclusión: El uso de un modelo de colgajos locales en una superficie convexa es efectivo para desarrollar habilidades básicas de colgajos locales en alumnos de medicina.

\section{ABSTRACT}

Introduction: Local flaps are a widely used surgical tool for the repair of primary defects. Most of the training models have been made on flat structures; however, their application in non-uniform structures requires prior training. Objective: To evaluate the performance of medical students in the acquisition of local flap techniques in a simulated model of the scalp. Material and methods: Nonrandomized experimental study. We designed a simulation model of pig skin mounted on a human skull. The training program consisted of two sessions. Each participant was videotaped performing an advancement and a rotation flap before and after the training. Then they trained the advancement and rotation flaps on the model. Student performance was evaluated according to OSATS guideline. Statistical analysis was performed with non-parametric statistics. Results: 21 participants were included. All participants significantly increased their OSATS scores when comparing the initial with the final evaluation both in advancement flap (15 [range 13-16] vs 23 [range 22-24] $p<0.001$ ), and in rotation flap (15 [range 14-17] vs 24 [range 23-25] $p<0.001)$. Conclusion: The use of a model of local flaps is effective in developing basic skills of local flaps of rotation and advancement in medical students.

\section{INTRODUCCIÓN}

$\mathrm{L}$ os colgajos locales son técnicas que cobran cada vez más importancia en la práctica quirúrgica, pues son utilizados en las distintas especialidades para cubrir defectos cutáneos o mucosos de difícil afrontamiento. Sin embargo, requieren entrenamiento previo para lograr un adecuado resultado estético y funcional adecuado. ${ }^{1}$ El modelo tradicional de enseñanza consistía en aprender los procedimientos directamente en pacientes reales. No obstante, hoy existen alternativas más seguras para que los alumnos realicen las primeras etapas de su aprendizaje, como el entrenamiento simulado. ${ }^{2,3}$ La simulación permite generar actividades estandarizadas, reproducibles y en un ambiente seguro. ${ }^{4}$ Diversos autores han evaluado el uso de modelos simulados para el aprendizaje de suturas y colgajos locales tanto en modelos sintéticos ${ }^{5-9}$ como en materiales ex vivo. ${ }^{10-20}$ Sin embargo,

Citar como: Pozo P, Inzunza M, Tejos R, Navia A, Jarry C, Achurra P, et al. Entrenamiento de colgajos locales en un modelo simulado de alta fidelidad y bajo costo. Simulación Clínica. 2020; 2 (3): 93-98. https://dx.doi.org/10.35366/97899 
la principal limitación de estos trabajos es que la mayoría se limitan a superficies planas. Si bien algunos trabajos han utilizado superficies cóncavas o convexas, 7,10,14 éstos se limitan a presentar el modelo de entrenamiento y no evalúan concretamente el desempeño de los alumnos.

El objetivo de este estudio es evaluar el desempeño de alumnos de medicina en la adquisición de técnicas de colgajos locales mediante un modelo simulado de cuero cabelludo en una superficie convexa.

\section{MATERIAL Y MÉTODOS}

\section{Participantes}

Se invitó a participar a alumnos de medicina sin experiencia previa en colgajos locales. Se incluyeron alumnos de cuarto y quinto año de medicina de nuestra institución, que cursaron y aprobaron previamente un curso electivo de habilidades básicas de sutura, previamente validado. ${ }^{21}$ Se excluyeron alumnos que refirieron participación previa en algún programa formal de colgajos locales.

\section{Modelo de entrenamiento}

La elaboración del modelo de entrenamiento de colgajos locales se realizó mediante metodología
Delphi modificada con panel de expertos sobre los modelos disponibles y alternativas de sostén para la piel. ${ }^{22}$ El consenso final fue un modelo de piel de cerdo ex vivo sobre un cráneo humano, denominado "modelo de entrenamiento convexo de colgajos locales".

\section{Programa de entrenamiento}

Se realizaron dos sesiones de entrenamiento de 90 minutos cada una. En la primera sesión se realizó una clase magistral sobre contenido teórico de técnicas de sutura y conceptos básicos de colgajos locales de rotación y avance. Posteriormente se realizó la evaluación inicial (ver sección Evaluación). En la segunda sesión del programa, los alumnos realizaron el entrenamiento en parejas, alternando el uso del modelo de simulación de colgajos locales. El entrenamiento consistió en el diseño y ejecución de dos colgajos locales distintos: colgajo de rotación y de avance. Un experto realizó retroalimentación directa a cada alumno durante la sesión de entrenamiento. Al término del programa de entrenamiento se realizó la evaluación final.

\section{Evaluación}

La evaluación inicial consistió en una videograbación de cada alumno diseñando y ejecutando

\begin{tabular}{|c|c|c|c|c|c|}
\hline Puntuación & Respeto por el tejido & Tiempo y movimientos & Uso del instrumento & $\begin{array}{l}\text { Tiempo de cirugía y } \\
\text { planificación posterior }\end{array}$ & Conocimientos \\
\hline 1 & $\begin{array}{l}\text { Con frecuencia, uso de } \\
\text { fuerza innecesaria sobre } \\
\text { el tejido o causa daños } \\
\text { por el uso inadecuado } \\
\text { de instrumentos }\end{array}$ & $\begin{array}{l}\text { Muchos movimientos } \\
\text { innecesarios }\end{array}$ & $\begin{array}{l}\text { Repetidamente } \\
\text { realiza movimientos } \\
\text { tentativos o torpes con } \\
\text { instrumentos }\end{array}$ & $\begin{array}{l}\text { Con frecuencia deja } \\
\text { de operar o necesita } \\
\text { discutir el próximo } \\
\text { movimiento }\end{array}$ & $\begin{array}{l}\text { Conocimientos } \\
\text { deficientes. Necesita } \\
\text { instrucción específica } \\
\text { en la mayoría de los } \\
\text { pasos a seguir }\end{array}$ \\
\hline 2 & Intermedio & Intermedio & Intermedio & Intermedio & Intermedio \\
\hline 3 & $\begin{array}{l}\text { Manejo cuidadoso } \\
\text { de los tejidos, pero } \\
\text { a veces causa daños } \\
\text { inadvertidos }\end{array}$ & $\begin{array}{l}\text { Uso eficiente de tiempo/ } \\
\text { movimiento, pero } \\
\text { algunos movimientos } \\
\text { inecesarios }\end{array}$ & $\begin{array}{l}\text { Uso competente de los } \\
\text { instrumentos, aunque de } \\
\text { vez en cuando duda o se } \\
\text { enreda con torpeza }\end{array}$ & $\begin{array}{l}\text { Capacidad demostrada } \\
\text { para la planificación, } \\
\text { progresión constante } \\
\text { de la intervención } \\
\text { quirúrgica }\end{array}$ & $\begin{array}{l}\text { Conocía todos los } \\
\text { aspectos importantes de } \\
\text { la operación }\end{array}$ \\
\hline 4 & Intermedio & Intermedio & Intermedio & Intermedio & Intermedio \\
\hline 5 & $\begin{array}{l}\text { De forma consistente } \\
\text { meneja adecuadamente } \\
\text { los tejidos con un daño } \\
\text { mínimo }\end{array}$ & $\begin{array}{l}\text { Economía de } \\
\text { movimientos con } \\
\text { eficiencia máxima }\end{array}$ & $\begin{array}{l}\text { Fluidez en el } \\
\text { movimiento de los } \\
\text { instrumentos sin torpeza }\end{array}$ & $\begin{array}{l}\text { Cada movimiento fue } \\
\text { obviamente planeado, } \\
\text { con un flujo sin esfuerzo } \\
\text { de un movimiento a otro }\end{array}$ & $\begin{array}{l}\text { Demostró familiaridad } \\
\text { demostrada con todos } \\
\text { los aspectos importantes } \\
\text { de la operación }\end{array}$ \\
\hline
\end{tabular}




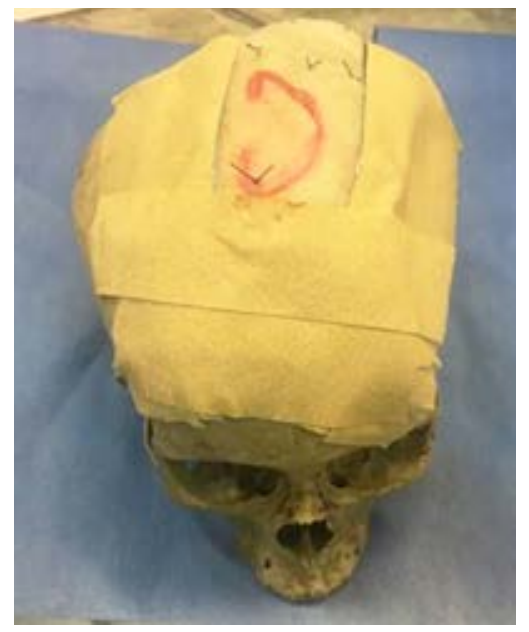

Figura 1: Modelo para colgajo de rotación.

un colgajo de avance y de rotación en el modelo de entrenamiento creado. Las videograbaciones fueron anónimas y se dio un tiempo máximo de 15 minutos para completar la tarea. La evaluación final se realizó al término de la segunda sesión de entrenamiento y fue idéntica a la inicial.

Luego, un experto, ciego a los alumnos y a la temporalidad de las grabaciones, evaluó aleatoriamente los videos. Este experto no participó en ninguna otra etapa del proyecto. Se asignaron puntajes de desempeño utilizando la escala global OSATS (objective structured assessment of technical skills), diseñada y validada para objetivar competencias técnicas (Tabla 1). ${ }^{19}$ Esta herramienta de evaluación está compuesta por cinco ítems con puntaje del 1 al 5, por lo que el puntaje mínimo es 5 y el máximo 25. Se determinó el número de participantes que excedieron el tiempo máximo de ejecución de la técnica. Se consideró como logro de autonomía del procedimiento un puntaje OSATS mayor o igual a 22 en cada técnica. ${ }^{23}$

\section{Evaluación de percepción}

Se aplicó una encuesta de percepción validada a los participantes. ${ }^{24}$ Ésta se realizó de forma anónima vía e-mail, y consistió en cinco preguntas tipo Likert sobre su percepción respecto al modelo de entrenamiento.

\section{Análisis estadístico}

El análisis estadístico se realizó con pruebas no paramétricas para comparación. Se utilizó el test de Wilcoxon para analizar diferencias entre la evaluación inicial y la final (análisis de muestras relacionadas). Se consideró un valor $p<0.05$ como estadísticamente significativo.

\section{RESULTADOS}

\section{Modelo de entrenamiento}

Se realizó un total de tres encuestas para llegar a un consenso final. El tejido seleccionado fue el de piel de cerdo ex vivo, dada su similitud con el cuero cabelludo, bajo costo y fácil acceso para los investigadores. El panel de expertos decidió utilizar un cráneo humano ex vivo obtenido del laboratorio de anatomía para la estructura de sostén para la piel. Éste fue seleccionado dado su bajo costo, fácil disponibilidad y alta fidelidad. La piel fue adherida al cráneo mediante telas adhesivas. La combinación de estos elementos permitió generar una estructura tridimensional que simula el cuero cabelludo (Figuras 1 a 3).

\section{Participantes}

Se incluyó un total de 21 participantes. La mediana de edad fue 22 años (rango 21-25). Un total de 11 (52\%) de los alumnos fueron de sexo masculino. No existieron pérdidas en el seguimiento, por lo que todos los alumnos fueron incluidos en el análisis.

\section{Evaluación de habilidades}

Todos los participantes realizaron la evaluación inicial y la evaluación final para ambas técnicas de colgajo. La mediana de puntuación OSATS para la evaluación inicial en colgajo de avance fue 15 (rango 13-16), mientras que para el colgajo de

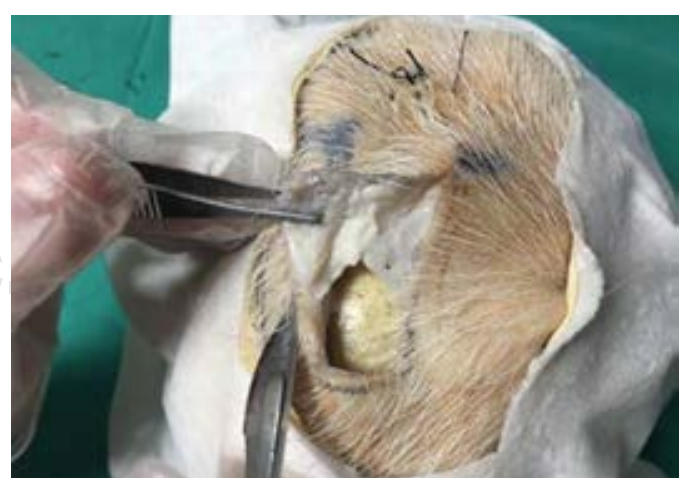

Figura 2: Disección de la piel del tejido subcutáneo en colgajo de avance. 
A

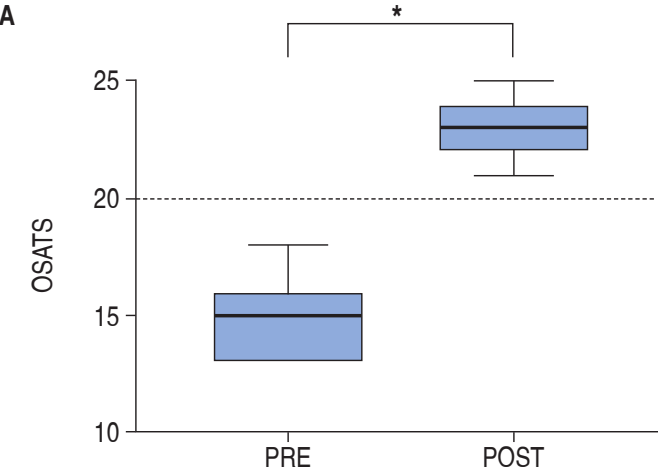

B

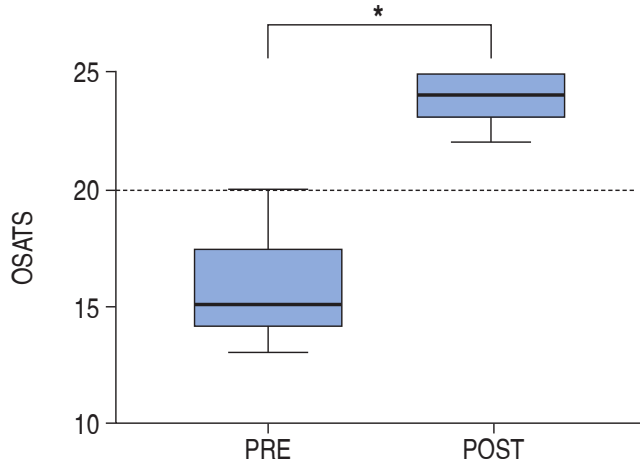

Figura 3: A) Puntaje OSATS previo y posterior a la evaluación en colgajo de avance. B) Puntaje OSATS previo y posterior a la evaluación en colgajo de rotación. *Diferencia estadisticamente significativa $(p<0.05)$. Linea punteada: puntaje OSATS mínimo para logro de autonomía. ${ }^{20}$

rotación fue 15 (rango 14-17). La mediana de puntuación OSATS para la evaluación final de colgajo de avance fue 23 (rango 22-24), y para el colgajo de rotación fue 24 (rango 23-25).

El tiempo máximo de desempeño de 15 minutos fue excedido por cinco alumnos en la evaluación inicial del colgajo de avance y por tres alumnos en el de rotación. En cambio, en la evaluación final de ambos colgajos ningún alumno excedió el tiempo máximo establecido.

Al comparar los resultados obtenidos en ambas evaluaciones, se observó que el puntaje OSATS de la evaluación final fue estadísticamente superior a la evaluación inicial en ambas técnicas (Figura 3). Asimismo, todos los participantes obtuvieron puntajes superiores al mínimo establecido para el logro de autonomía.

\section{Percepción de la actividad}

La encuesta de percepción fue respondida por todos los participantes. En términos generales, la mayoría de los estudiantes valoró positivamente la experiencia.

Sobre la incisión con bisturí en la piel, 81\% evaluó el modelo como "fiel a la realidad quirúrgica" y el 19\% restante como "medianamente parecido a la realidad quirúrgica". En cuanto a la disección de la piel sobre el tejido subcutáneo, $58 \%$ lo califica como "fiel a la realidad quirúrgica", $35 \%$ como "intermedio" y $7 \%$ como "poco parecido a la realidad quirúrgica". Al preguntar por el diseño y avance del colgajo, $86 \%$ lo define como "fiel a la realidad quirúrgica" y $14 \%$ "intermedio". Acerca del paso de puntos a través de la piel, $42 \%$ lo valora como "fiel a la realidad quirúrgica", $50 \%$ como "intermedio" y $8 \%$ restante como "poco fiel a la realidad quirúrgica". Por último, $77 \%$ prefiere utilizar el modelo de piel de cerdo sobre $23 \%$ que opta por no utilizarlo.

\section{DISCUSIÓN}

Nuestro estudio propone el uso de un modelo ex vivo convexo, de alta fidelidad para la práctica de colgajos locales. Todos los participantes mejoraron significativamente sus habilidades al comparar la evaluación inicial con la final. Es decir, el entrenamiento en este modelo fue efectivo para la adquisición de habilidades en colgajos locales. Además, la mayoría de los participantes valoró positivamente la experiencia.

Las principales fortalezas de este trabajo radican en la creación de un modelo de colgajos de alta fidelidad y la elaboración de un programa de entrenamiento evaluado para determinar que el modelo es útil para enseñar habilidades técnicas. La fidelidad proporcionada por el uso de un cráneo humano es muy alta, ya que esta estructura de sostén es idéntica al cráneo de un paciente vivo. Adicionalmente, la piel de cerdo utilizada también proporciona un alto índice de fidelidad, puesto que se ha descrito como una estructura similar al cuero cabelludo de los humanos. ${ }^{25}$ Otra de las fortalezas de este estudio es acercar a los alumnos de medicina a las técnicas quirúrgicas de colgajos locales. Los alumnos de medicina tienen poca exposición a técnicas quirúrgicas básicas y avanzadas, principalmente debido al gran contenido de medicina general que deben abordar en un tiempo acotado de formación. ${ }^{26-29}$ Si bien los colgajos locales son realizados comúnmente por cirujanos, cada vez son utilizados con más frecuencia por otros profesionales médicos, ya que pueden ser de gran utilidad en defectos cutáneos o mucosos de difícil afrontamiento. ${ }^{20}$ 
Los materiales sintéticos también se han utilizado previamente como modelos de menor fidelidad para el entrenamiento de colgajos locales. ${ }^{5-9}$ Schwartz y colaboradores describieron el uso de yeso para recrear la cara, cubierto con varias láminas de apósitos adhesivos que representan las diferentes capas de tejidos de la cara. ${ }^{8,9}$ Nicolaou y su equipo utilizaron un maniquí cubierto de múltiples capas de silicona para la simulación de colgajos bilobulares y romboidales. ${ }^{9}$ Si bien estos modelos pueden ser económicos, tienen la desventaja de que la flexibilidad y resistencia de estos tejidos difieren de las características de una piel real.

Otros autores también han utilizado materiales ex vivo para la elaboración de modelos de colgajos locales. ${ }^{10-18}$ Hassan y colegas ${ }^{10}$ utilizaron la piel de cerdo sobrepuesta en una cabeza de un maniquí humano de plástico. El mecanismo de sujeción de la piel a la cabeza del maniquí fueron clavos en distintos puntos estratégicos. Si bien este trabajo es similar a lo propuesto por nuestro equipo, sus principales falencias son el fácil desplazamiento de la piel sobre el maniquí y la ausencia de una adecuada validación del modelo, ya que sólo se limitaron a presentar sus características y no demostraron con pruebas objetivas su utilidad para la adquisición de habilidades. Otro estudio publicado por Bauer y su grupo $^{20}$ utiliza una cabeza de cerdo ex vivo para enseñar a estudiantes de medicina sobre el uso de distintos tipos de colgajos. Si bien Bauer y sus colegas obtuvieron buenos resultados de aprendizaje, consideramos que la utilización de una cabeza completa de cerdo tiene las desventajas de incrementar los costos, dificultad de almacenamiento y consideraciones de bioseguridad adicionales a lo que representa el uso exclusivo de un segmento de piel.

Durante la evaluación de la percepción, los alumnos describieron que se debe mejorar la disección del tejido y el paso de puntos. A pesar de estas limitaciones, $77 \%$ de los alumnos prefirió el modelo de piel de cerdo previo a la experiencia con pacientes. Dadas las preferencias de los alumnos por este modelo, creemos que una solución para sus sugerencias podría ser el uso de piel de cerdo de un segmento corporal distinto, en donde la piel sea más delgada. Ejemplo de esto podría ser la piel del área abdominal del cerdo.

Una de las limitaciones de este estudio fue la falta de disponibilidad de modelos adicionales para el entrenamiento individual. Si bien esto puede afectar negativamente por la disminución de exposición de cada alumno al entrenamiento, también creemos que los beneficia al generar una instancia de entrenamiento y retroalimentación por pares. ${ }^{21} \mathrm{Al}$ compartir el modelo, necesariamente deben observar a su compañero ejecutar la tarea, lo que puede favorecer el aprendizaje al identificar los errores y aciertos del otro participante. ${ }^{30}$

Una de las interrogantes que surgen de este trabajo es la efectividad de estas sesiones de entrenamiento en la práctica con pacientes reales. Por esta razón, creemos que se deben realizar estudios que evalúen la transferencia de habilidades desde el modelo simulado a la práctica clínica real.

\section{CONCLUSIÓN}

El uso de un modelo de colgajos utilizando tejido ex vivo de piel de cerdo sobre un cráneo humano es efectivo para la adquisición de habilidades iniciales en colgajos locales de rotación y avance en estudiantes de medicina.

\section{REFERENCIAS}

1. Hashimoto I, Abe Y, Ishida S, et al. Development of skin flaps for reconstructive surgery: random pattern flap to perforator flap. J Med Invest. 2016; 63: 159-162.

2. Minha S, Shefet D, Sagi D, et al. "See one, sim one, do one"- a national pre-internship boot-camp to ensure a safer "student to doctor" Transition. PLoS One. 2016; 11 (3): e0150122.

3. Aggarwal R, Darzi A. Technical-skills training in the 21st century. N Engl J Med. 2006; 355: 2695-2696.

4. Okuda Y, Bryson EO, DeMaria S Jr, et al. The utility of simulation in medical education: what is the evidence? Mt Sinai J Med. 2009; 76 (4): 330-343.

5. Denadai R, Kirylko L. Teaching basic plastic surgical skills on an alternative synthetic bench model. Aesthet Surg J. 2013; 33 (3): 458-461.

6. Liew SH, McPhail J, Morton JD, et al. A non-animal facial model for teaching local flaps to trainees. $\mathrm{Br}$ J Plast Surg. 2004; 57: 374-375.

7. Davis CR, Fell M, Khan U. Facial reconstruction using a skull and foam training model. J Plast Reconstr Aesthet Surg. 2014; 67: 126-127.

8. Schwarz K, Davison SP. Periumbilical full-thickness skin graft donor site for pretibial skin cancer excisions. Plast Reconstr Surg. 2008; 121: 232e-233e.

9. Nicolaou M, Yang GZ, Darzi A, Butler PE. An inexpensive 3 -D model for teaching local flap design on the face and head. Ann R Coll Surg Engl. 2006; 88 (3): 320.

10. Hassan Z, Hogg F, Graham K. A 3-dimensional model for teaching local flaps using porcine skin. Ann Plast Surg. 2014; 73: 362-363.

11. Ibrahim N, Chauhan I, Varma S. A novel method for practising local skin flaps. Ann R Coll Surg Engl. 2018; 100 (4): 338.

12. Chan WY, Dalal M. Cost-effective plastic surgery skills training. J Plast Reconstr Aesthet Surg. 2010; 63: e136-e137; author reply e138. 
13. Esteban D, Fraga MF, Shimba LG, et al. Basic plastic surgery training using human skin. Plast Reconstr Surg. 2009; 123: 90e-92e.

14. Iqbal A, Ellabban MG. The dynamic tension real tissue training model for local flap design training. Plast Reconstr Surg. 2005; 115: 1434-1436.

15. Wanzel KR, Matsumoto ED, Hamstra SJ, Anastakis DJ. Teaching technical skills: training on a simple, inexpensive, and portable model. Plast Reconstr Surg. 2002; 109: 258-263.

16. Isaacson DS, Edmonds PR, Isaacson G. The galliform (Turkey thigh) model for resident training in facial plastic surgery. Laryngoscope. 2014; 124: 866-868.

17. Turhan-Haktanir N, Sancaktar N. Useful material for skin flap training for inexperienced residents. J Plast Reconstr Aesthet Surg. 2007; 60: 1169-1170.

18. Iqbal A, Ellabban MG, Srivastava S, Jaffe W. Discard little, learn more: the real tissue training model. Plast Reconstr Surg. 2005; 115: 948-949.

19. Martin JA, Regehr G, Reznick R, et al. Objective structured assessment of technical skill (OSATS) for surgical residents. Br J Surg. 1997; 84 (2): 273-278.

20. Bauer F, Koerdt S, Rommel N, et al. Reconstruction of facial defects with local flaps--a training model for medical students? Head Face Med. 2015; 11: 30.

21. Tejos R, Crovari F, Achurra P, et al. Video-based guided simulation without peer or expert feedback is not enough: a randomized controlled trial of simulationbased training for medical students. World J Surg. 2020.

22. Ávila CM, Masdeu Ávila C. Metodología Delphi en salud. Hipertens Riesgo Vasc. 2015; 32: 12-16.

23. Carlson J, Tomkowiak J, Knott P. Simulation-based examinations in physician assistant education: A comparison of two standard-setting methods. J Physician Assist Educ. 2010; 21: 7-14.

24. Villagrán I, Tejos R, Chahuan J, et al. Percepción de estudiantes de pregrado de Medicina de talleres de simulación de procedimientos médico-quirúrgicos. Rev Méd Chile. 2018; 146: 786-795.

25. Panse N, Panse S, Kulkarni P, et al. Awareness and perception of plastic surgery among healthcare professionals in Pune, India: do they really know what we do? Plast Surg Int. 2012; 2012: 962169.

26. Burd A, Chiu T, McNaught C. Plastic surgery in the undergraduate curriculum: the importance of considering students' perceptions. Br J Plast Surg. 2004; 57 (8): 773-779.

27. Antoszewski B, Kardas P, Kasielska A, Fijalkowska M. Family physicians' perception of plastic surgery and its influence on referral. A survey from Poland. Eur J Gen Pract. 2012; 18 (1): 22-225.

28. Dunkin CS, Pleat JM, Jones SA, Goodacre TE. Perception and reality-a study of public and professional perceptions of plastic surgery. Br J Plast Surg. 2003; 56 (5): 437-443.

29. Al-Nuaimi Y, McGrouther G, Bayat A. Modernizing medical careers in the UK and plastic surgery as a possible career choice: undergraduate opinions. J Plast Reconstr Aesthet Surg. 2006; 59 (12): 1472-1474.

30. González LR, Molina ZH, García-Huidobro DM, Stevens MP, Jadue TA, Riquelme UA, et al. Tutoría por pares de distinto año académico en la enseñanza de habilidades quirúrgicas básicas en estudiantes de pregrado de medicina. Rev Cir. 2019; 71 (6): 545-551.

\section{Correspondencia:}

\section{Dra. Susana Searle}

Profesor asistente

Sección de Cirugía Plástica y Reconstructiva.

División de Cirugía, Pontificia Universidad

Católica de Chile.

Diagonal Paraguay 362,

Santiago, Chile, 8330077.

E-mail: plasticsurgeryuc2@uc.cl 